\title{
Neutron scattering from a coordination polymer quantum paramagnet
}

\author{
T. Hong, ${ }^{1}$ M. Kenzelmann, ${ }^{1,2}$ M. M. Turnbull,${ }^{3}$ C. P. Landee ${ }^{3}$ B. D. Lewis,${ }^{3}$ \\ K. P. Schmidt, ${ }^{4}$ G. S. Uhrig, ${ }^{5}$ Y. Qiu ${ }^{2,6}$ C. Broholm,${ }^{1,2}$ and D. H. Reich ${ }^{1}$ \\ ${ }^{1}$ Department of Physics and Astronomy, The Johns Hopkins University, Baltimore, Maryland 21218, USA \\ ${ }^{2}$ National Institute of Standards and Technology, Gaithersburg, Maryland 20899, USA \\ ${ }^{3}$ Carlson School of Chemistry and Department of Physics, \\ Clark University, Worcester, Massachusetts 01610, USA \\ ${ }^{4}$ Institute of Theoretical Physics, École Polytechnique Fédérale de Lausanne, CH 1015 Lausanne, Switzerland \\ ${ }^{5}$ Theoretische Physik, FR 7.1, Geb. 38, Universität des Saarlandes, 66123 Saarbrücken, Germany \\ ${ }^{6}$ Department of Materials and Engineering, University of Maryland, College Park, Maryland 20742, USA
}

(Dated: September 14, 2018)

\begin{abstract}
Inelastic neutron scattering measurements are reported for a powder sample of the spin-1/2 quantum paramagnet $\mathrm{Cu}$ (Quinoxaline) $\mathrm{Br}_{2}$. Magnetic neutron scattering is identified above an energy gap of $1.9 \mathrm{meV}$. Analysis of the sharp spectral maximum at the onset indicates that the material is magnetically quasi-one-dimensional. Consideration of the wave vector dependence of the scattering and polymeric structure further identifies the material as a two-legged spin- $1 / 2$ ladder. Detailed comparison of the data to various models of magnetism in this material based on the single mode approximation and the continuous unitary transformation are presented. The latter theory provides an excellent account of the data with leg exchange $J_{\|}=2.0 \mathrm{meV}$ and rung exchange $J_{\perp}=3.3 \mathrm{meV}$.
\end{abstract}

PACS numbers: 75.10.Jm, 75.40.Gb, 75.50.Ee

\section{INTRODUCTION}

Progress towards understanding the cooperative quantum physics of one dimensional systems is frequently gated by the availability of suitable model systems for experiments. While oxides offer the possibility of carrier doping, coordination polymer magnets have energy scales that are well suited for high magnetic field experiments that traverse phase boundaries. Such experiments have been important for elucidating the fermionic nature of low energy excitations in the uniform spin- $1 / 2$ chain ${ }^{1}$ and their remarkable transition to a gapped solitonic spectrum upon application of an effective staggered field $?^{2}$

A natural next step in the experimental exploration of one dimensional spin- $1 / 2$ systems is to examine magnetic excitations as a function of field and temperature in two legged spin- $1 / 2$ ladders. The Hamiltonian of this system has the form

$$
\begin{aligned}
H & =\sum_{i} J_{\perp} \mathbf{S}_{1, i} \cdot \mathbf{S}_{2, i} \\
& +J_{\|}\left(\mathbf{S}_{1, i} \cdot \mathbf{S}_{1, i+1}+\mathbf{S}_{2, i} \cdot \mathbf{S}_{2, i+1}\right),
\end{aligned}
$$

where $i$ indexes rungs and 1,2 each leg as illustrated in Fig. 1. The Lieb-Schultz-Mattis theorem ${ }^{3}$ allows for a finite gap in the excitation spectrum and indeed analytical, $\stackrel{4.5,6,7.8,9.10}{,}$ numerical,$\stackrel{4}{,}$ and experimental ${ }^{11}$ work has confirmed that a spin gap does exist. A key experiment is to close this spin gap with an external field and explore the predicted quantum critical high field phase ${ }^{12}$ A rich zero field excitation spectrum that should feature both bound states and continua also remains to be examined experimentally ${ }^{10,13}$
Unfortunately as of now no experimental model system has been identified to enable neutron scattering experiment in the high field phase. $(\mathrm{La}, \mathrm{Sr}, \mathrm{Ca}){ }_{14} \mathrm{Cu}_{24} \mathrm{O}_{41} 14$ and $\mathrm{SrCu}_{2} \mathrm{O}_{3} 11$ do appear to be spin ladders but the high energy scales for these systems render them unsuitable for the proposed experiments. The energy scales for $\mathrm{Cu}_{2}(1,4 \text { - diazacycloheptane })_{2} \mathrm{Cl}_{4}(\mathrm{CuHpCl})^{15 \cdot 16.17 .18 .19}$ and $(\mathrm{VO})_{2} \mathrm{P}_{2} \mathrm{O}_{\tau}{ }^{20.21}$ are appropriate and they were previously identified as spin- $1 / 2$ ladders on the basis of specific heat and magnetization measurements. However, further investigations with neutron scattering revealed that the exchange interactions in these materials do not correspond to spin ladders despite spin ladder structural motifs. In the case of $\mathrm{CuHpCl}$ there were early indications from magnetization measurements that the material is not one dimensional and subsequent neutron scattering experiments showed that the group velocity for magnetic excitation has no substantial anisotropy 22 In addition, the molecular bond, which was thought to be the rung of the ladder, assumes a frustrated configuration in the ground state. Hence $\mathrm{CuHpCl}$ is now thought to have a complex frustration induced singlet ground state ${ }^{22}$ $(\mathrm{VO})_{2} \mathrm{P}_{2} \mathrm{O}_{7}$ on the other hand is quasi-one-dimensional but rather than being a spin ladder, neutron scattering experiments showed that it is an alternating spin chain with the chain direction perpendicular to the putative ladder direction. ${ }^{23,24.25}$

These false starts illustrate that bulk measurements, while sensitive to the magnetic density of states, can not provide definite conclusions regarding the geometry of interacting spin system with a singlet ground state. With the goal of identifying spin ladder systems for high field experiments, we have initiated a project to synthesize potential coordination polymer spin ladders and subject them to inelastic neutron scattering experiments for 


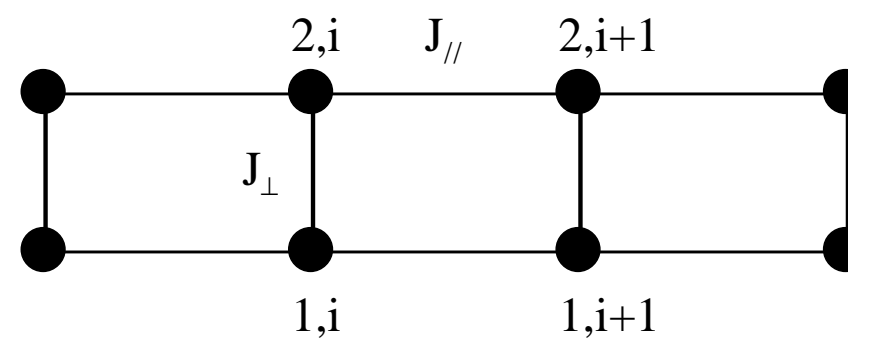

FIG. 1: Schematic of a two-leg spin ladder system, indica1 ing the rung interaction $J_{\perp}$ and the rail interaction $J_{\|}$. Th indexing of spin sites used in Eq. (I) is also shown.

verification. $\mathrm{Cu}$ (Quinoxaline) $\mathrm{Br}_{2}$ is a first out of ser eral such candidate materials, ${ }^{26}$ Susceptibility measure ments on this material27 are well described by a spir ladder model with antiferromagnetic exchange constant $J_{\|}=2.61 \mathrm{meV}$ and $J_{\perp}=3.02 \mathrm{meV}$. Magnetizatio measurements 27 show a phase transition from a nor magnetic singlet ground state to a magnetized state nea a critical field $H_{c}=14 \mathrm{~T}$, indicating the presence c an energy gap $\Delta=g \mu_{B} H_{c} \approx 1.7 \mathrm{meV}$. Furthermor the square root singular onset of magnetization abov the critical field suggests that the system is magneticall one-dimensional.

In the present paper, we report inelastic neutron scat tering experiment on a deuterated powder sample c $\mathrm{Cu}$ (Quinoxaline) $\mathrm{Br}_{2}$ to determine whether this materic is indeed a spin ladder and to establish the strength of th relevant exchange interactions. The comparison of wav vector and energy dependent magnetic neutron scatte1ing data to comprehensive model calculations carried out as a function of rung and leg exchange constants strongly indicate that $\mathrm{Cu}$ (Quinoxaline) $\mathrm{Br}_{2}$ is a coordination polymer spin ladder, with weak inter-ladder interactions. Final verification will require inelastic scattering experiments from single crystals.

The crystal structure of $\mathrm{Cu}$ (Quinoxaline) $\mathrm{Br}_{2}$ $\left(\mathrm{Cu}\left(\mathrm{C}_{8} \mathrm{H}_{6} \mathrm{~N}_{2}\right) \mathrm{Br}_{2}\right)$ is monoclinic with space group $\mathrm{C} 2 / \mathrm{m}$ and lattice constants $\mathrm{a}=13.1745(15) \AA, \mathrm{b}=6.9293(8)$ $\AA, \mathrm{c}=10.3564(12) \AA$, and $\beta=107.699(2)^{\circ} .27$ Quinoxaline $\left(\mathrm{C}_{8} \mathrm{H}_{6} \mathrm{~N}_{2}\right)$ has a tendency towards polymeric structures where quinoxaline forms a bridge between metal atoms $\stackrel{28}{2} \mathrm{In} \mathrm{Cu}$ (Quinoxaline) $\mathrm{Br}_{2}, \mathrm{Cu}_{2} \mathrm{Br}_{4}$ dimers are linked to adjacent dimers by bridging quinoxaline molecules along the monoclinic $\mathbf{b}$ axis as shown in Fig. 2(a). The inter-dimer spacing along the $\mathbf{b}$-axis is 6.929 A. Hydrogen bond mediated interactions may exist between molecular units displaced by $\mathbf{d}_{\mathbf{1}}=(\mathbf{a} \pm \mathbf{b}) / \mathbf{2}$ and $\mathbf{d}_{\mathbf{2}}=\mathbf{c}$ (See Fig. 22). If there were sufficiently strong, the material could be a two-dimensional bi-layer system

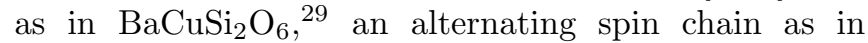
$\mathrm{Cu}\left(\mathrm{NO}_{3}\right)_{2} \cdot 2.5 \mathrm{D}_{2} \mathrm{O} \stackrel{30}{=}$ or a three dimensional system of interacting spin pairs as in $\mathrm{TlCuCl}_{3}{ }^{31}$ and $\mathrm{KCuCl}_{3} 3^{32.33}$

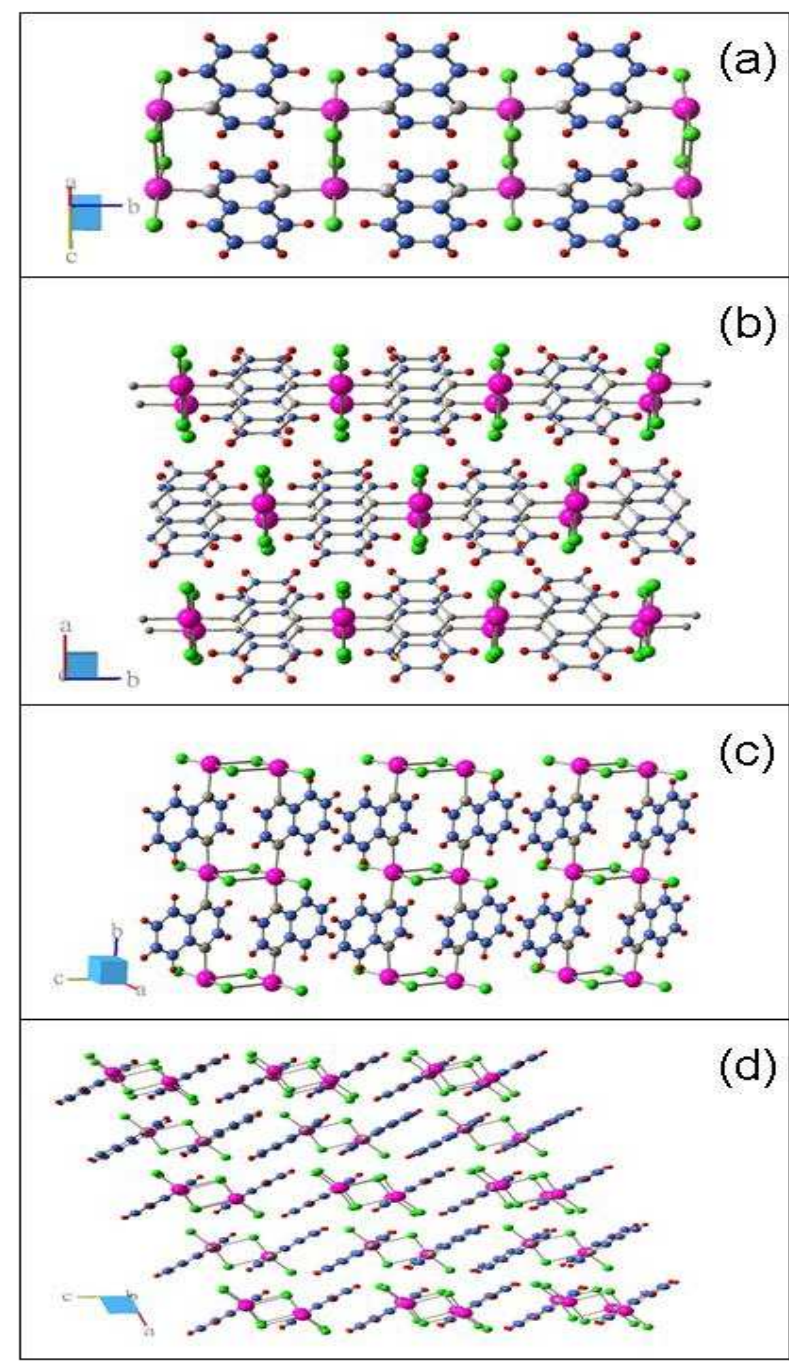

FIG. 2: Structure of $\mathrm{Cu}$ (Quinoxaline) $\mathrm{Br}_{2}$ projected along four different directions to illustrate potential interacting spin models. (a) The molecular ladder structure of $\mathrm{Cu}$ (Quinoxaline) $\mathrm{Br}_{2}{ }^{27}$, which the ladder extends along the monoclinic $\mathbf{b}$ axis. Interactions along the $(\mathbf{a} \pm \mathbf{b}) / \mathbf{2}$ and $\mathbf{c}$ directions could lead to two dimensional dimer models in the $\mathbf{a}-\mathbf{b}$ or b-c planes (frame (b) and (c)) or a three dimensional dimer model (frame (d)). Color coding is as follows: Pink: $\mathrm{Cu}$, Green:Br, Blue:C, Red: H, and Grey:N.

\section{EXPERIMENTAL TECHNIQUES}

\section{A. Sample and Neutron Instrumentation}

The neutron scattering sample consisted of 27 grams of deuterated powder in an annular aluminum can with inner diameter $2.42 \mathrm{~cm}$, outer diameter $2.92 \mathrm{~cm}$, and height $10 \mathrm{~cm}$. The powder was obtained through precipitation of a 1:1 molar solution of anhydrous $\mathrm{CuBr}_{2}$ and deuterated d-4 quinoxaline dissolved in a small amount of $95 \%$ ethanol. Prompt gamma neutron activation analysis and NMR measurements showed that $58 \%$ of the hydrogen 
sites in our sample were occupied by deuterium. This is less than the $2 / 3$ deuteration level of the $\mathrm{d}-4$ quinoxaline starting material indicating some hydrogen/deuterium exchange with the solvent.

Inelastic neutron scattering measurements were performed using the disk chopper time-of-flight spectrometer (DCS) $)^{34}$ at the National Institute of Standards and Technology (NIST) Center for Neutron Research in Gaithersburg, Maryland. A disk chopper system was used to select a $167 \mathrm{~Hz}$ pulsed neutron beam with an energy of $4.87 \mathrm{meV}$ and a pulse width of $79 \mu$ s from the NCNR cold neutron source. The 0.65 steradian detection system probed inelastic scattering with energy transfer $-4.17 \mathrm{meV} \leq \hbar \omega \leq 3.84 \mathrm{meV}$ and momentum transfer $0.13 \AA^{-1} \leq \bar{Q} \leq 3.40 \AA^{-1}$. The full width at half maximum (FWHM) elastic energy resolution was $\delta \hbar \omega \simeq 0.18$ $\mathrm{meV}$.

\section{B. Neutron Scattering Data Analysis}

The remnant hydrogen in the sample gives rise to a scattering cross section of 201 barn per formula unit compared to a total magnetic scattering cross section from $\mathrm{Cu}^{2+}$ of 0.146 barn per formula unit. There are several distinct challenges associated with the strong incoherent nuclear scattering. (1) The strong elastic scattering cross section reveals tails of the energy resolution function that can dwarf the magnetic signal well beyond the FWHM of the resolution function. (2) Phonon scattering and multiple scattering processes involving phonons produce a strong background even at low temperatures that must be determined accurately in order to isolate the magnetic scattering. (3) Both magnetic and phonon scattering are frequently preceded or followed by incoherent elastic nuclear scattering. Because incoherent scattering is approximately wave vector independent this leads to a $Q$-averaged contribution of both types of scattering at any wave vector. We have developed accurate techniques for dealing with each of these issues and because they are essential for this research they are described in the following sections.

\section{Temperature independent background}

The elastic background from the tails of the resolution function can be determined by utilizing the fact that inelastic scattering obeys the principle of detailed balance whereas elastic scattering at sufficiently low temperatures, to a good approximation, can be approximated as being temperature independent. Under these circumstances the raw measured count rate, $I_{r}(\omega, T)$, for any specific value of wave vector transfer satisfies:

$$
\begin{aligned}
I_{r}(\omega, T) & =B(\omega)+I(\omega, T) . \\
I_{r}(-|\omega|, T) & =B(-|\omega|)+I(|\omega|, T) \exp (-\hbar|\omega| / k T) .(3)
\end{aligned}
$$

Here $B(\omega)$ is the temperature independent background and $I(\omega, T)$ represents all inelastic scattering processes that satisfy detailed balance at the temperature $T$. An underlying assumption here is that resolution effects can be neglected for the inelastic scattering. By measuring data at two different temperatures $T_{1}=1.4 \mathrm{~K}$ and $T_{2}=60$ $\mathrm{K}$ and using equations (2) and (3) it is possible to extract values for $B(|\omega|), B(-|\omega|), I\left(\omega, T_{1}\right)$ and $I\left(\omega, T_{2}\right)$ from the corresponding four equations.

\section{Subtracting phonon scattering}

The above procedure isolates inelastic scattering from temperature independent elastic scattering and detector dark current. However, this scattering intensity still has contributions from magnetic as well as phonon scattering. To remove the low temperature contribution from phonon scattering, $I_{p}\left(\omega, T_{1}\right)$, and hence isolate low temperature magnetic scattering, $I_{m}\left(\omega, T_{1}\right)$, we use the fact that phonon scattering in hydrogenous systems dwarfs magnetic scattering for $k_{B} T>>\hbar \omega$. Hence we make the following approximation for $k_{B} T_{2}>\hbar \omega$ :

$$
I\left(\omega, T_{2}\right) \equiv I_{p}\left(\omega, T_{2}\right)+I_{m}\left(\omega, T_{2}\right) \approx I_{p}\left(\omega, T_{2}\right) .
$$

We further assume that one phonon scattering events dominate over multi-phonon events and we neglect the temperature dependence of the Debye Waller factor such that ${ }^{35}$

$$
I_{p}\left(\omega, T_{1}\right) \approx \frac{1-\exp \left(-\beta_{2} \hbar \omega\right)}{1-\exp \left(-\beta_{1} \hbar \omega\right)} I\left(\omega, T_{2}\right) .
$$

The final expression for the magnetic inelastic scattering as deduced from the measurements at $T_{1}=1.5 \mathrm{~K}$ and $T_{2}=60 \mathrm{~K}$ is therefore

$$
I_{m}\left(\omega, T_{1}\right) \approx I\left(\omega, T_{1}\right)-\frac{1-\exp \left(-\beta_{2} \hbar \omega\right)}{1-\exp \left(-\beta_{1} \hbar \omega\right)} I\left(\omega, T_{2}\right) .
$$

\section{Multiple Scattering}

Multiple scattering is difficult to avoid when probing weak magnetic scattering in the presence of strong incoherent scattering. Here we describe how to account for the dominant double scattering process that involves incoherent elastic scattering preceded or followed by a weaker inelastic process. For simplicity we assume that the incoherent elastic scattering is isotropic and independent of neutron energy and we neglect anisotropy introduced by the sample geometry 36 Under those circumstances the incoherent scattering event in a double scattering process effectively randomizes the direction of scattering for the preceding or subsequent inelastic scattering:

$$
\begin{aligned}
I^{\prime}(Q, \omega)= & \mathcal{T} I(Q, \omega) \\
& +(1-\mathcal{T}) \int_{\left|k_{i}-k_{f}\right|}^{k_{i}+k_{f}} I\left(Q^{\prime}, \omega\right) \frac{Q^{\prime} d Q^{\prime}}{2 k_{i} k_{f}} .
\end{aligned}
$$




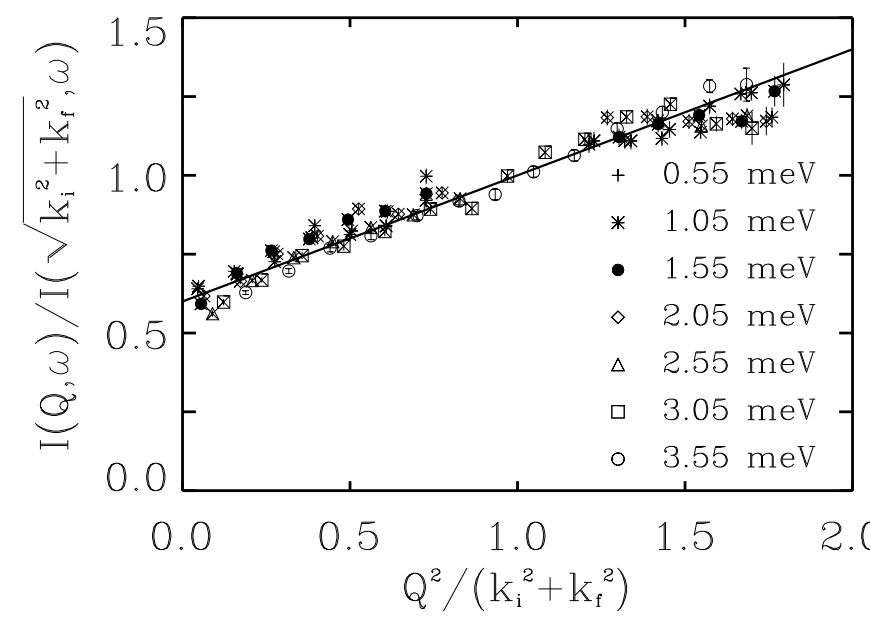

FIG. 3: Scaled wave vector dependence of inelastic neutron scattering from $\mathrm{Cu}$ (Quinoxaline) $\mathrm{Br}_{2}$ at $T=60 \mathrm{~K}$ and for various values of energy transfer. The scaling behavior is consistent with double scattering by phonons and incoherent elastic nuclear scattering as described by Eq. (7) and (8).

Here $k_{i}$ and $k_{f}$ are the incident and scattered wave vectors respectively. The integral implements the average over scattering directions for the inelastic process. $\mathcal{T}$ is an "effective" sample transmission that can be determined through analysis of phonon scattering. Approximating the intensity of single event phonon scattering for a thin sample as $I_{p}(Q, \omega)=f(\omega) Q^{2}$ we find that

$$
I_{p}^{\prime}(Q, \omega)=f(\omega)\left(k_{i}^{2}+k_{f}^{2}\right)\left(\mathcal{T} \frac{Q^{2}}{k_{i}^{2}+k_{f}^{2}}+(1-\mathcal{T})\right) .
$$

Fig. 3] shows phonon scattering intensity at $T=60 \mathrm{~K}$ as a function of $Q^{2} /\left(k_{i}^{2}+k_{f}^{2}\right)$. Data for several values of energy transfer, $\hbar \omega$, scale in agreement with Eq. (8) and yield an effective value of $\mathcal{T}=0.40$. For comparison the average transmission through a spherical version of our sample as calculated considering only incoherent scattering is 0.30. In the following we shall use Eq. 7 with $\mathcal{T}=0.40$ when comparing the measured inelastic magnetic scattering, $I_{m}(\omega, T)$, to theoretical models of spin dynamics in $\mathrm{Cu}$ (Quinoxaline) $\mathrm{Br}_{2}$.

\section{Normalized magnetic scattering}

Anisotropic self shielding effects associated with the annular sample geometry were taken into account using a numerical integration technique $\frac{37}{}$ Absolute normalization of the data was subsequently achieved using elastic incoherent scattering from the sample, duly considering the hydrogen/deuterium ratio determined through neutron activation and NMR analysis ${ }^{19}$ The technique is estimated to be accurate to within $20 \%$. The normalized magnetic scattering intensity, $\tilde{I}_{m}$, is related to the resolution smeared dynamic spin correlation function as follows:

$$
\begin{aligned}
\tilde{I}_{m}(Q, \omega)= & 2 \int d Q^{\prime} \hbar d \omega^{\prime} \mathcal{R}_{Q \omega}\left(Q-Q^{\prime}, \omega-\omega^{\prime}\right) \\
& \times\left|\frac{g}{2} F\left(\mathbf{Q}^{\prime}\right)\right|^{2} \widetilde{\mathcal{S}}\left(Q^{\prime}, \omega^{\prime}\right) .
\end{aligned}
$$

Here $\mathcal{R}_{Q \omega}\left(Q-Q^{\prime}, \omega-\omega^{\prime}\right)$ is a unity normalized resolution function that is peaked on the scale of the FWHM resolution for $Q \approx Q^{\prime}$ and $\hbar \omega \approx \hbar \omega^{\prime}, g \simeq 2.12$ is the Landé g-factor for $\mathrm{Cu}^{2+}$ in $\mathrm{Cu}$ (Quinoxaline) $\mathrm{Br}_{2}{ }^{27} F(\mathbf{Q})$ is the anisotropic magnetic form factor appropriate for a hole in the $3 d_{x^{2}-y^{2}}$ orbital ${ }^{38}$ Based on bond distances and their coplanarity it appears that the two short $\mathrm{Cu}-\mathrm{Br}$ bonds and the two bonds to the neighboring quinoxaline molecules define the $\hat{\mathbf{x}}-\hat{\mathbf{y}}$ plane for the $3 d_{x^{2}-y^{2}}$ copper orbitals. We used this assumption to model the effects of the anisotropic magnetic form factor. The spherical average of the dynamic spin correlation function is

$$
\widetilde{\mathcal{S}}(Q, \omega)=\int \frac{d \Omega_{\hat{Q}}}{4 \pi} \frac{1}{2} \sum_{\alpha \beta}\left(\delta_{\alpha \beta}-\hat{Q}_{\alpha} \hat{Q}_{\beta}\right) \mathcal{S}^{\alpha \beta}(\mathbf{Q}, \omega),
$$

and the dynamic spin correlation function proper is

$$
\begin{aligned}
\mathcal{S}^{\alpha \beta}(\mathbf{Q}, \omega)= & \frac{1}{2 \pi \hbar} \int d t e^{i \omega t} \frac{1}{N} \sum_{\mathbf{R R}^{\prime}} \\
& <S_{\mathbf{R}}^{\alpha}(t) S_{\mathbf{R}^{\prime}}^{\beta}(0)>e^{-i \mathbf{Q} \cdot\left(\mathbf{R}-\mathbf{R}^{\prime}\right)} .
\end{aligned}
$$

Unless specifically stated, we use the notation defined in S. M. Lovesey's book on thermal neutron scattering, 35

\section{EXPERIMENTAL RESULTS}

Fig. 四 (a-b) shows raw Q-integrated data at $\mathrm{T}=60 \mathrm{~K}$ and $\mathrm{T}=1.4 \mathrm{~K}$. The range covered is $0.5 \AA^{-1} \leq \mathrm{Q} \leq 1.0$ $\AA^{-1}$. The solid symbols in Fig. 目(b) show $B(\omega)$ as determined by Eq. (2) and Eq. (3). It is clear from this analysis that the strong incoherent elastic scattering from hydrogen produces intense tails of scattering intensity well beyond the $0.18 \mathrm{meV}$ FWHM of the resolution function. The increase of the count rate beyond $3 \mathrm{meV}$ (see

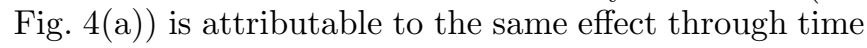
of flight frame overlap.

A small peak is visible in the raw low temperature data (Fig. 4(b)) at approximately $2 \mathrm{meV}$. Following background subtraction, Fig. प(d) clearly shows that this peak marks the onset of a continuum of scattering in the wave vector integrated spectrum. Fig. 4(c) shows that this continuum is not present at $\mathrm{T}=60 \mathrm{~K}$ where as expected, thermally activated phonon scattering dominates. The solid line in Fig. 4(d) shows the projected contribution from phonon scattering at $1.4 \mathrm{~K}$ as derived from the data in Fig. 4(c) using Eq. (5). While incoherent inelastic phonon scattering produces a background contribution that increases with $\hbar \omega$, it is apparent that the bounded continuum above a $\sim 2 \mathrm{meV}$ spectral gap is 


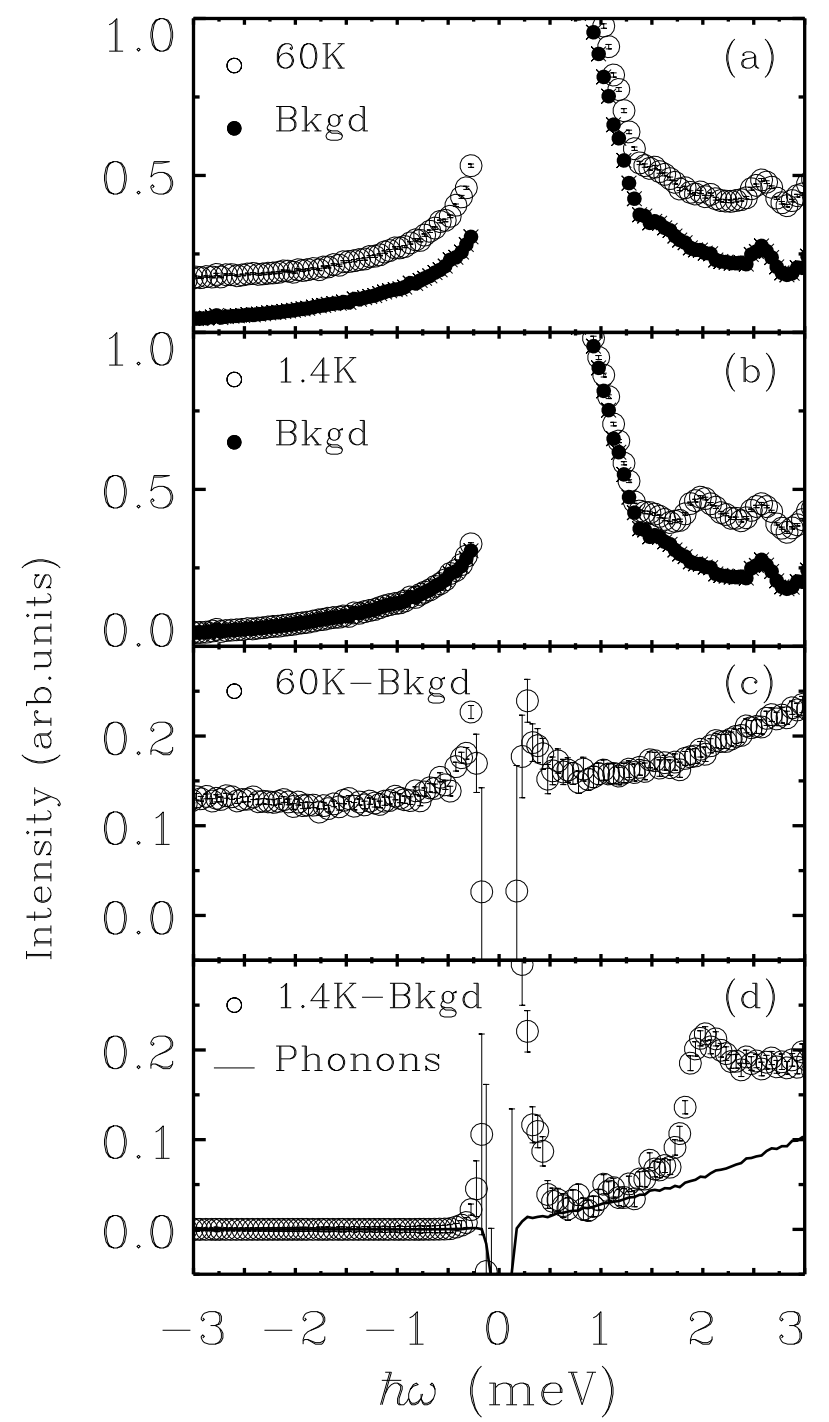

FIG. 4: The energy dependence of Q-integrated neutron scattering from $\mathrm{Cu}$ (Quinoxaline) $\mathrm{Br}_{2}$ at (a) $\mathrm{T}=60 \mathrm{~K}$ and (b) $\mathrm{T}=1.4$ $\mathrm{K}$ before detailed balance correction. In both frames solid symbols show the temperature independent background determined as described in section Ш.B,1. Background subtracted data at (c) $\mathrm{T}=60 \mathrm{~K}$ and (d) $\mathrm{T}=1.4 \mathrm{~K}$ data. The solid line is phonon scattering contribution at $\mathrm{T}=1.4 \mathrm{~K}$ as determined by Eq. (5).

associated with inelastic magnetic scattering. This spingap is consistent with the activation energy derived from bulk measurements. ${ }^{27}$

Having verified that magnetic scattering can be isolated from incoherent nuclear scattering through the techniques described in section $\amalg$ the analysis can now be replicated as a function of wave vector transfer. The results are summarized in Figs. [5] and [6] where the data have furthermore been normalized to report values for $\widetilde{I}(Q, \omega)$ as defined by Eq. (9).

Fig. [5 shows the normalized scattering intensity

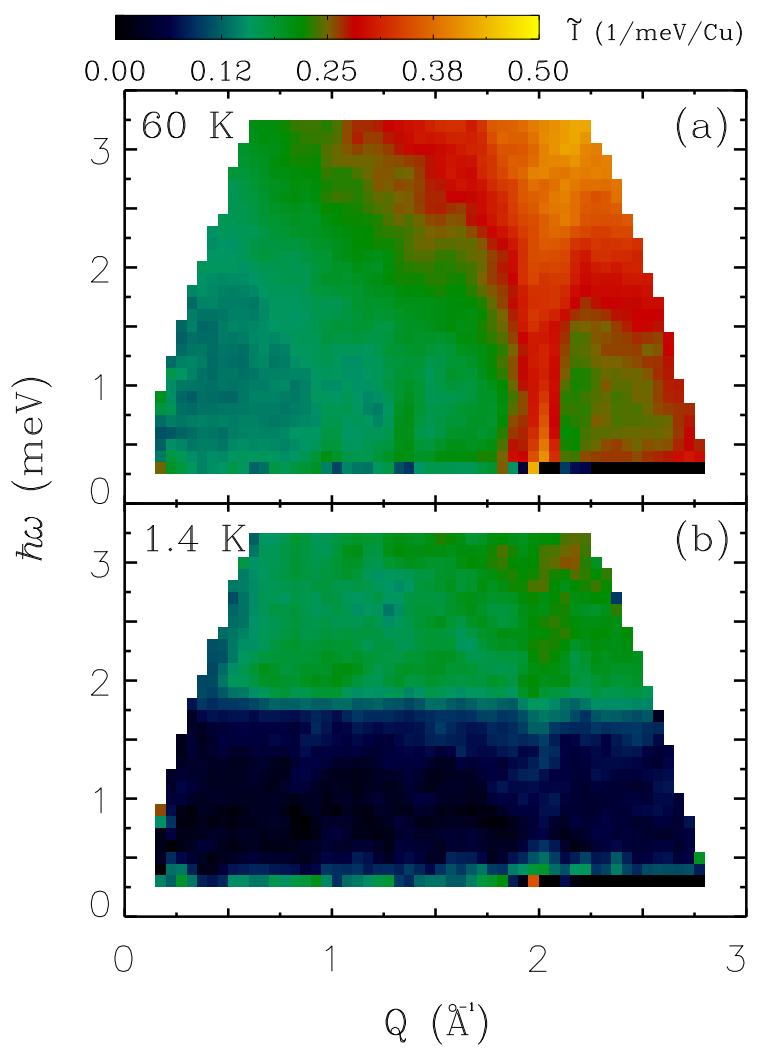

FIG. 5: Normalized powder inelastic neutron scattering intensity for $\mathrm{Cu}$ (Quinoxaline) $\mathrm{Br}_{2}$ at (a) $\mathrm{T}=60 \mathrm{~K}$ and (b) $\mathrm{T}=1.4 \mathrm{~K}$ after detailed balance correction as described in section $\amalg . B, 1$.

$\widetilde{I}(Q, \omega)$ for $\mathrm{Cu}$ (Quinoxaline) $\mathrm{Br}_{2}$ at $\mathrm{T}=1.4 \mathrm{~K}$ and $\mathrm{T}=60$ $\mathrm{K}$ after subtracting the temperature independent background determined through Eq. (2) and Eq. (3). At $\mathrm{T}=60 \mathrm{~K}$ the scattering increases with wave vector transfer as expected for phonon scattering. Further analysis of the $Q$-dependence of these data was provided in section [B]3. At low temperatures there is a clear onset of scattering for $\hbar \omega>2 \mathrm{meV}$ and this is consistent with magnetic neutron scattering from a quantum paramagnet. However, a growth in the scattering intensity towards higher $Q$ confirms the conclusions from the previous spectral analysis that even the low $T$ scattering contains significant contributions from phonon scattering.

Fig. [6) shows the phonon contribution to low temperature scattering derived from the $\mathrm{T}=60 \mathrm{~K}$ data based on Eq. (5). Subtracting these data from the data in Fig. (5) concludes the process of isolating the $Q$ and $\hbar \omega$ dependent magnetic scattering. From these data, which are displayed in Fig. 6 (b), it is apparent that there is a sharp onset of magnetic scattering for $\hbar \omega \simeq 1.9 \mathrm{meV}$ with a finite- $Q$ maximum. In the following we shall show that these are features of a quasi-one-dimensional antiferromagnet with a singlet ground state and that the data furthermore are consistent with a spin ladder extending along the $\mathbf{b}$-axis. 


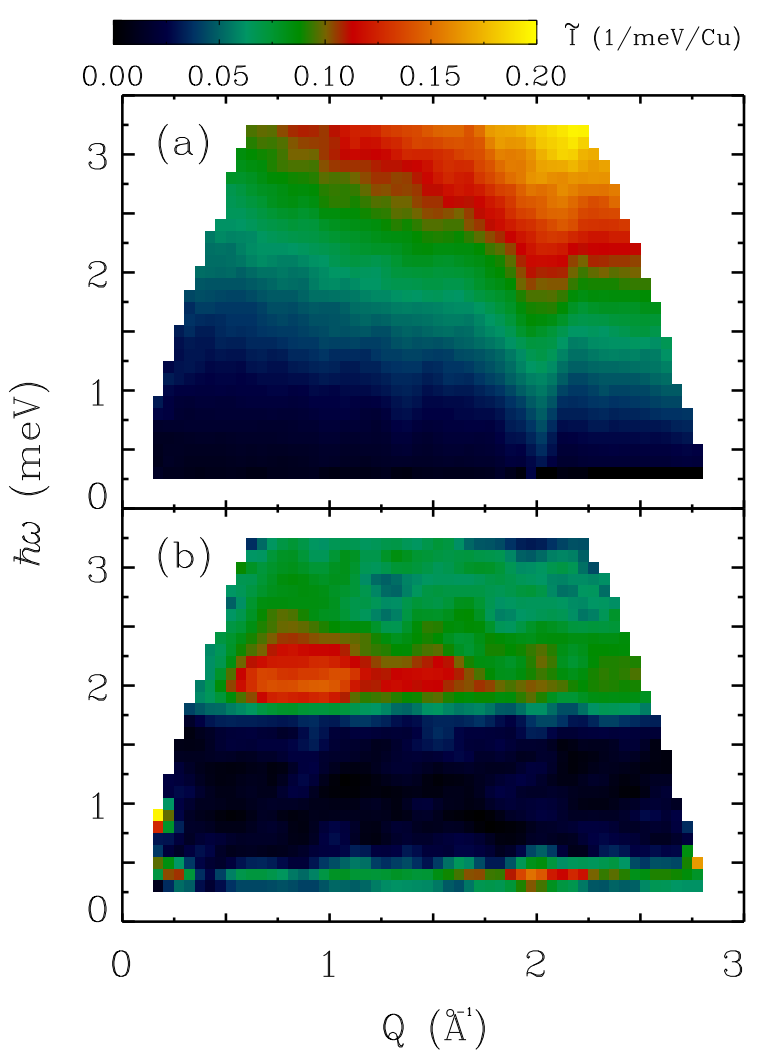

FIG. 6: Normalized inelastic scattering intensity for $\mathrm{Cu}$ (Quinoxaline) $\mathrm{Br}_{2}$ (a) Phonon scattering intensity at $\mathrm{T}=1.4$ $\mathrm{K}$. (b) Magnetic scattering intensity at $\mathrm{T}=1.4 \mathrm{~K}$ after subtracting the phonon contribution as described in section Ш.B,1.

\section{ANALYSIS AND DISCUSSION}

\section{A. Single Mode Approximation}

For most but not all ${ }^{39}$ quantum magnets, the dominant spectral weight resides in a resonant mode. When this excitation is between a singlet ground state and an excited triplet without magnetic long range order, it is called a triplon ${ }^{40}$ We prefer to reserve the common term magnon for systems with magnetic long range order. The "single mode approximation" (SMA) provides an excellent account of the dynamic spin correlation function for a dominant triplon. The starting point is the following low temperature approximation for $\mathcal{S}(\mathbf{Q}, \omega)$, which concentrates all spectral weight in a resonant mode.

$$
\mathcal{S}^{\alpha \beta}(\mathbf{Q}, \omega)=\mathcal{S}(\mathbf{Q}) \delta(\hbar \omega-\epsilon(\mathbf{Q})) \delta_{\alpha \beta} .
$$

The first moment sum-rule ${ }^{41}$ links the equal time correlation function, $\mathcal{S}(\mathbf{Q})$, to the dispersion relation as follows

$$
\mathcal{S}(\mathbf{Q})=-\frac{2}{3} \frac{1}{\epsilon(\mathbf{Q})}\left[J_{\mathbf{d}}\left\langle\mathbf{S}_{1, i} \cdot \mathbf{S}_{2, i}\right\rangle(1-\cos (\mathbf{Q} \cdot \mathbf{d}))\right]
$$

Here we make the simplifying approximation that the molecular bond with displacement vector $\mathbf{d}$ dominates the structure factor. Within this framework, we examine different models of magnetism in $\mathrm{Cu}$ (Quinoxaline) $\mathrm{Br}_{2}$ by varying parameters in the following phenomenological dispersion relation that allows for inter-molecular interactions along the $(\mathbf{a} \pm \mathbf{b}) / \mathbf{2}$ and $\mathbf{c}$ directions:

$$
\begin{aligned}
\epsilon(\mathbf{Q})=\epsilon_{\mathbf{b}}(k) & +2 B_{h k} \cos 2 \pi h \cdot \cos 2 \pi k \\
& +B_{l} \cos (2 \pi l)
\end{aligned}
$$

Here $\mathbf{Q}=h \mathbf{a}^{*}+k \mathbf{b}^{*}+l \mathbf{c}^{*}$. For models that approach the spin ladder limit we use the following strong rung coupling perturbation expansion,, 42

$$
\begin{aligned}
\epsilon_{\mathbf{b}}(k)= & J_{\perp}\left[1+\frac{J_{\|}}{J_{\perp}} \cos 2 \pi k+\frac{1}{4}\left(\frac{J_{\|}}{J_{\perp}}\right)^{2}(3-\cos 4 \pi k)\right. \\
& -\frac{1}{8}\left(\frac{J_{\|}}{J_{\perp}}\right)^{3}(2 \cos 2 \pi k+2 \cos 4 \pi k-\cos 6 \pi k-3) \\
& \left.+O\left(\frac{J_{\|}}{J_{\perp}}\right)^{4}\right],
\end{aligned}
$$

For models where the dispersion along $\mathbf{b}$ is not dominant we use the simpler form

$$
\epsilon_{\mathbf{b}}(k)=B_{0}+B_{k} \cos 2 \pi k
$$

The spherical average of $\mathcal{S}(\mathbf{Q}, \omega)$ (Eq. (10) was in general calculated numerically except for truly onedimensional models where we used the analytical result 19 .

\section{B. One-Triplon CUT Model}

For an ab-initio description of the dynamic spin correlation function for the two-leg ladder, we use a particle conserving Continuous Unitary Transformation (CUT) $10,43,44,45$ The CUT is realized in a perturbative fashion about the limit of isolated rung dimers and can yield the spectral weight and dispersion of the triplon elementary excitations, as well as the multi-triplon contributions. Here we are interested in the low-energy part of the dynamical structure factor for $x=J_{\|} / J_{\perp} \leq 1$. In this regime the dynamical structure factor is dominated by the one-triplon part. Two-triplon contributions are located at higher energies and carry only a small part of the total spectral weight $(10 \%$ for $x=0.6)$. It is thus justified to restrict the discussion to the one-triplon part of the dynamical structure factor, which is characterized by the one-triplon dispersion $\epsilon_{\mathrm{CUT}}(\mathbf{Q})$ and the one-triplon spectral weight $a^{2}(\mathbf{Q})$. The high-order series for both quantities are expressed in terms of an internal parameter of the system, the energy gap $\Delta$, instead of $x$, and quantitative results for $x \leq 1$ are obtained by extrapolation 46.47

The dynamic spin correlation function at $T=0$ and for $\omega>0$ is given by

$$
\begin{aligned}
\mathcal{S}_{\mathrm{CUT}}(\tilde{q}, \omega)= & -\frac{1}{\pi} \Im m \frac{a^{2}(\tilde{q})}{\hbar \omega-\epsilon_{\mathrm{CUT}}(\tilde{q})+i \delta} \\
& =\frac{1}{\pi} \frac{a^{2}(\tilde{q}) \delta}{\left(\hbar \omega-\epsilon_{\mathrm{CUT}}(\tilde{q})\right)^{2}+\delta^{2}} .
\end{aligned}
$$


Here $\delta=0.02 \mathrm{meV}$ was used to avoid divergences without introducing broadening beyond the experimental resolution. The spherical average of the dynamic spin correlation function is obtained as follows:

$$
\begin{aligned}
\widetilde{\mathcal{S}}_{\mathrm{CUT}}(Q, \omega) & =\frac{1}{4 \pi} \int_{0}^{\pi} \sin \theta d \theta \int_{0}^{2 \pi} d \phi \\
& \times 2 S_{\mathrm{CUT}}\left(Q_{\|} b, \omega\right)\left(1-\cos \left(d Q_{\perp} \sin \phi\right)\right) .
\end{aligned}
$$

The integral over $\phi$ can be computed analytically leading to

$$
\begin{aligned}
\widetilde{\mathcal{S}}_{\mathrm{CUT}}(Q, \omega) & =\frac{2}{b Q} \int_{0}^{Q u} d y S_{\mathrm{CUT}}(y, \omega) \\
& \times\left[1-J_{0}\left(\frac{d}{b} \sqrt{\left(b^{2} Q^{2}-y^{2}\right)}\right)\right]
\end{aligned}
$$

where $y=Q b \cos \theta$ and $J_{0}(x)$ is the zeroth order Bessel function. From the structure of the material, we have $d=3.75 \stackrel{\circ}{A}$ and $\mathrm{b}=6.93 \stackrel{\circ}{A}$.

\section{Comparison between theory and data}

We now compare each of these models of magnetism in $\mathrm{Cu}$ (Quinoxaline) $\mathrm{Br}_{2}$ to the observed magnetic scattering intensity. Multiple scattering involving inelastic magnetic scattering followed or preceded by incoherent elastic nuclear scattering was added to the calculated single event scattering as described by Eq. (7) with the experimentally determined effective transmission $\mathcal{T}=0.4$. We also allowed for an overall additive constant to account for any discrepancies in the background subtraction.

\section{1. $Q$ and $E$ cuts}

For an overview, Fig. 7] shows the energy dependence of the magnetic scattering averaged over wave-vectors from $0.5 \AA^{-1}$ to $1.0 \AA^{-1}$ as follows:

$$
\widetilde{I}(\omega)=\frac{\int Q^{2} \widetilde{I}(Q, \omega) d Q}{\int Q^{2} d Q},
$$

These data are a measure of the magnetic density of states and are sensitive to the dimensionality of the spin system. The dashed line in Fig. [7 shows a fit to the one dimensional CUT spectrum. The peak in the data is broader than this truly 1D model. The dotted line shows the spectrum for a two dimensional model where $B_{0}=3.82 \mathrm{meV}$ and $B_{h k}=1.02 \mathrm{meV}$, which produce a gap in the spectrum that is consistent with the data. This 2D model does not produce a peak at the spectral onset as observed in the experiment. A quasi-onedimensional model (solid line in Fig. (7) with $B_{0}=3.82$ $\mathrm{meV}, B_{k}=1.84 \mathrm{meV}, B_{h k}=0.08 \mathrm{meV}$ and $B_{l}=0.14$ $\mathrm{meV}$ on the other hand yields an acceptable fit. From this we may conclude that $\mathrm{Cu}$ (Quinoxaline) $\mathrm{Br}_{2}$ is magnetically quasi one-dimensional.

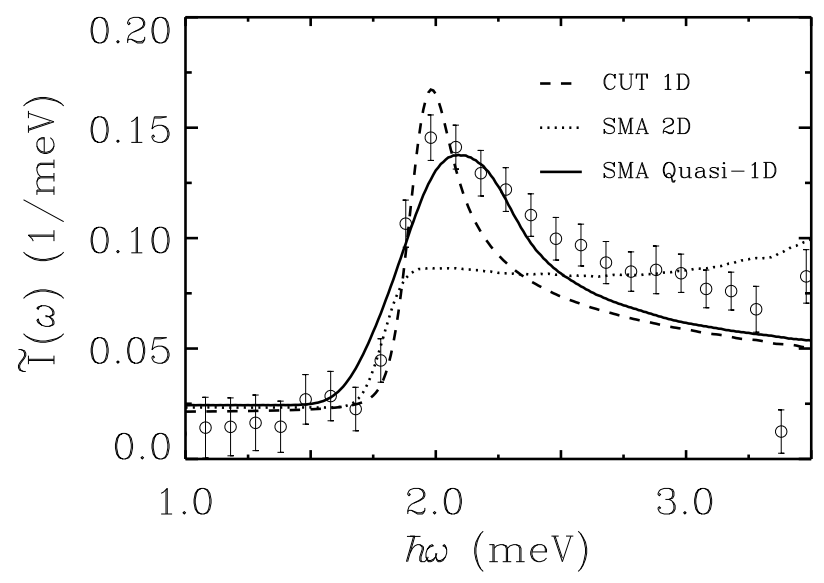

FIG. 7: Energy dependence of the normalized magnetic scattering intensity for $\mathrm{Cu}$ (quinoxaline) $\mathrm{Br}_{2}$ averaged over wave vectors $0.5 \AA^{-1} \leq \mathrm{Q} \leq 1.0 \AA^{-1}$. Open circles are experimental data. The dashed line indicates the one-triplon dynamic spin correlation function from the Continuous Unitary Transformation theory. The dotted line shows the spectrum for a two dimensional model with $B_{0}=3.82 \mathrm{meV}$ and $B_{h k}=1.02$ $\mathrm{meV}$. The solid line shows the spectrum for a quasi-onedimensional ladder model with $B_{0}=3.82 \mathrm{meV}, B_{k}=1.84$ $\mathrm{meV}, B_{h k}=0.08 \mathrm{meV}$, and $B_{l}=0.14 \mathrm{meV}$. All models were convolved with the experimental resolution function and an overall constant was fit to account for any discrepancies in the background subtraction procedure.

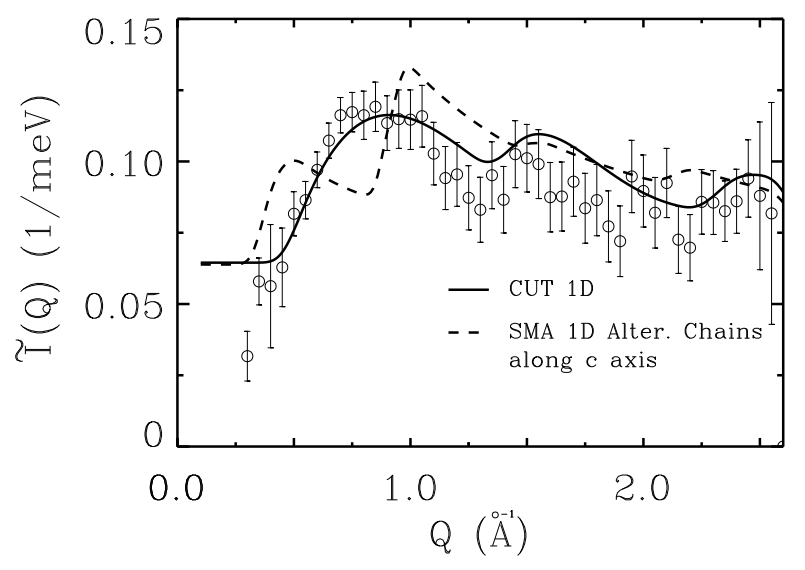

FIG. 8: Wave-vector dependence of normalized magnetic scattering intensity for $\mathrm{Cu}$ (quinoxaline) $\mathrm{Br}_{2}$ at $1.4 \mathrm{~K}$, integrated over energy transfers $1.85 \mathrm{meV} \leq \hbar \omega \leq 2.65 \mathrm{meV}$. Open circles are experimental data. The solid and dash lines indicate the dynamic spin correlation function of the one-triplon CUT and SMA 1D alternating spin chain models, respectively, convolved with the experimental resolution function. Multiple scattering was added to the models as calculated from Eq. (7) using the experimentally determined value of $\mathcal{T}=0.40$. Note that data for $Q<0.5 \AA^{-1}$ does not include the full energy range due to the kinematic limitations of the experiment (see Fig. [11]. 
To establish the relevant one dimensional model, we examine the wave-vector dependence of magnetic scattering averaged over the energy range from $1.85 \mathrm{meV}$ to $2.65 \mathrm{meV}$ (Fig. 8). $\widetilde{I}(Q)$ is sensitive to spatial spin correlations and in particular the intra-dimer spin spacing, $d$. The data show a rounded maximum at $Q_{0} \simeq 0.8 \AA^{-1}$, indicating that the spins forming the dominant correlated spin pairs are separated by $d \approx \pi / Q_{0}=4 \AA$. For comparison the nearest neighbor $\mathrm{Cu}-\mathrm{Cu}$ separation within the $\mathrm{Cu}_{2} \mathrm{Br}_{4}$ molecular unit is $3.75 \AA$. The solid line in Fig. 8 shows the CUT ladder model prediction. The excellent agreement with the data is strong support for the ladder model. Conversely the c-axis alternating chain model for one-dimensional magnetism in $\mathrm{Cu}$ (Quinoxaline) $\mathrm{Br}_{2}$ produces the dashed line which is clearly inconsistent with the data.

\section{Intensity contour maps and global fits}

We performed global fits of the dynamic spin correlation function associated with the models described in section IV to the $Q$ and $\omega$ dependent magnetic neutron scattering from $\mathrm{Cu}$ (Quinoxaline) $\mathrm{Br}_{2}$. The results are summarized in Figs. 7-11 A least-square fit of the ladder single-mode approximation gave $\left\langle\mathbf{S}_{1, i} \cdot \mathbf{S}_{2, i}\right\rangle=-0.37(1)$, $\left\langle\mathbf{S}_{1, i} \cdot \mathbf{S}_{1, i+1}\right\rangle=-0.04(1)$. The former value is close to the expectation value of $-3 / 8$ for a spin dimer singlet ground state. We also found $J_{\|}=1.98(4) \mathrm{meV}, J_{\perp}=3.05(1)$ meV. The ratio $J_{\|} / J_{\perp} \approx 0.65$ indicates that the perturbative expression for the dispersion relation in Eq. (16) is at the limit of applicability. Allowing for inter-ladder coupling through finite values for $B_{h k}$ and $B_{l}$ reduced the $\chi^{2}$ from 1.3 to 1.1 for $B_{h k}=0.08 \mathrm{meV}$ and $B_{l}=0.14$ meV, which indicates that there are weak interactions between ladders. Fig. 111(b) shows the best fit which corresponds to $B_{0}=3.82 \mathrm{meV}, B_{k}=1.84 \mathrm{meV}, B_{h k}=0.08$ $\mathrm{meV}, B_{l}=0.14 \mathrm{meV}$, and $J_{\mathbf{d}}\left\langle\mathbf{S}_{1, i} \cdot \mathbf{S}_{2, i}\right\rangle=-1.0 \mathrm{meV}$.

As opposed to the strong coupling perturbation expansion, the CUT can provide results for $\mathcal{S}(\mathrm{Q}, \omega)$ for $J_{\perp} \approx J_{\|}$. Fits were undertaken for fixed exchange ratios $x=J_{\|} / J_{\perp}$ ranging from 0.5 to 1 . For each value of $x$, the only adjustable parameters was an overall scale factor and the constant background. $J_{\perp}$ was selected so as to produce the observed value of $\Delta$ for each value of $x$ as listed in Table! Fig. 9 shows the $x$ dependent smallest possible value for the $\chi^{2}$ goodness of fit criterium. The best fit was obtained for $x=0.6$. The corresponding values of the exchanges constants are $J_{\|}=1.98 \mathrm{meV}$ and $J_{\perp}=3.3 \mathrm{meV}$. The entire powder averaged dynamic spin correlation function for this model is shown in Fig. 10 a). Fig. 111 (a) compares the CUT model calculations for $x=0.6$ to the experimental data. Fig. [10)(b) shows that the strong coupling dispersion relation obtained from the SMA fits is in fact also in good agreement with that derived from the CUT.

Whether the corresponding scattering cross section is approximated by the CUT or the SMA, the ladder model

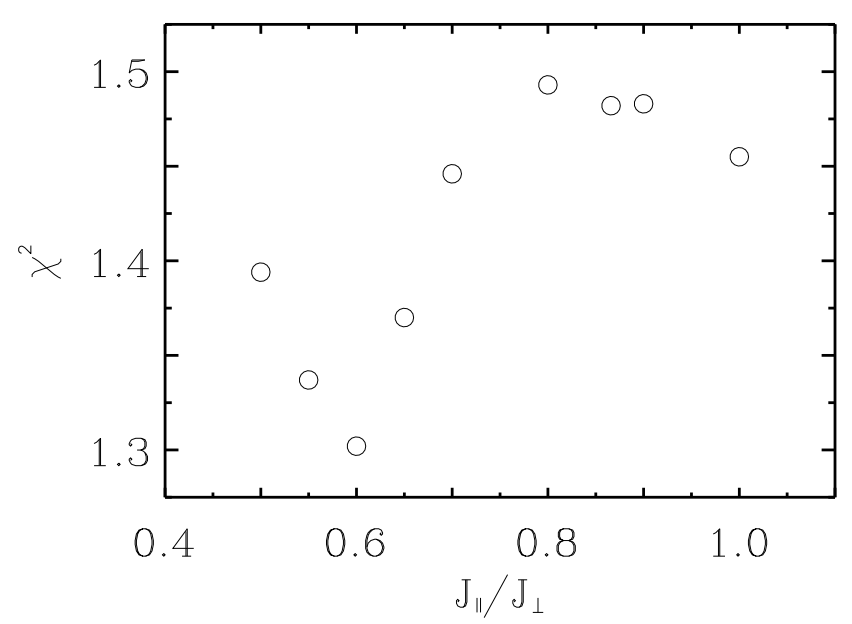

FIG. 9: $\chi^{2}=\frac{1}{N_{\text {free }}} \sum_{j}\left(I_{j}^{o b s}-I_{j}^{e x}\right)^{2} / \sigma_{j}^{2}$ versus the ratio of rail to rung exchange in the comparison of magnetic neutron scattering data for $\mathrm{Cu}$ (quinoxaline) $\mathrm{Br}_{2}$ to the Continuous Unitary Transformation theory of one-triplon excitations in a b-axis spin ladder.

TABLE I: Values of ladder exchange constants for which the one triplon contribution to $\widetilde{S}(Q, \omega)$ was calculated for comparison to the data

\begin{tabular}{lccc}
\hline \hline$J_{\|}(\mathrm{meV})$ & $J_{\perp}(\mathrm{meV})$ & $x=J_{\|} / J_{\perp}$ & $\chi^{2}$ \\
\hline 1.56 & 3.12 & 0.5 & 1.39 \\
1.77 & 3.21 & 0.55 & 1.34 \\
1.98 & 3.3 & 0.6 & 1.30 \\
2.22 & 3.41 & 0.65 & 1.37 \\
2.46 & 3.52 & 0.7 & 1.45 \\
2.96 & 3.70 & 0.8 & 1.49 \\
3.29 & 3.80 & 0.866 & 1.48 \\
3.47 & 3.85 & 0.9 & 1.48 \\
3.98 & 3.98 & 1.0 & 1.46 \\
\hline \hline
\end{tabular}

clearly provides an excellent account of the data. The virtue of CUT in this context is that apart from the exchange constants and an overall scale factor that in principle can be determined through the total moment sum rule, there are no adjustable parameters.

\section{CONCLUSION}

In summary, we used neutron scattering to determine the origin of quantum paramagnetism in $\mathrm{Cu}$ (Quinoxaline) $\mathrm{Br}_{2}$. The methods of detailed balance correction, phonon subtraction, and multiple scattering correction were described and used to extract the relatively weak magnetic signal from the strong nuclear incoherent scattering for quantitative comparison to theoretical models. The observed spin gap $\Delta \approx 1.9 \mathrm{meV}$ is consistent with magnetic susceptibility and high-field magnetization measurement. ${ }^{27}$ The neutron scattering data 


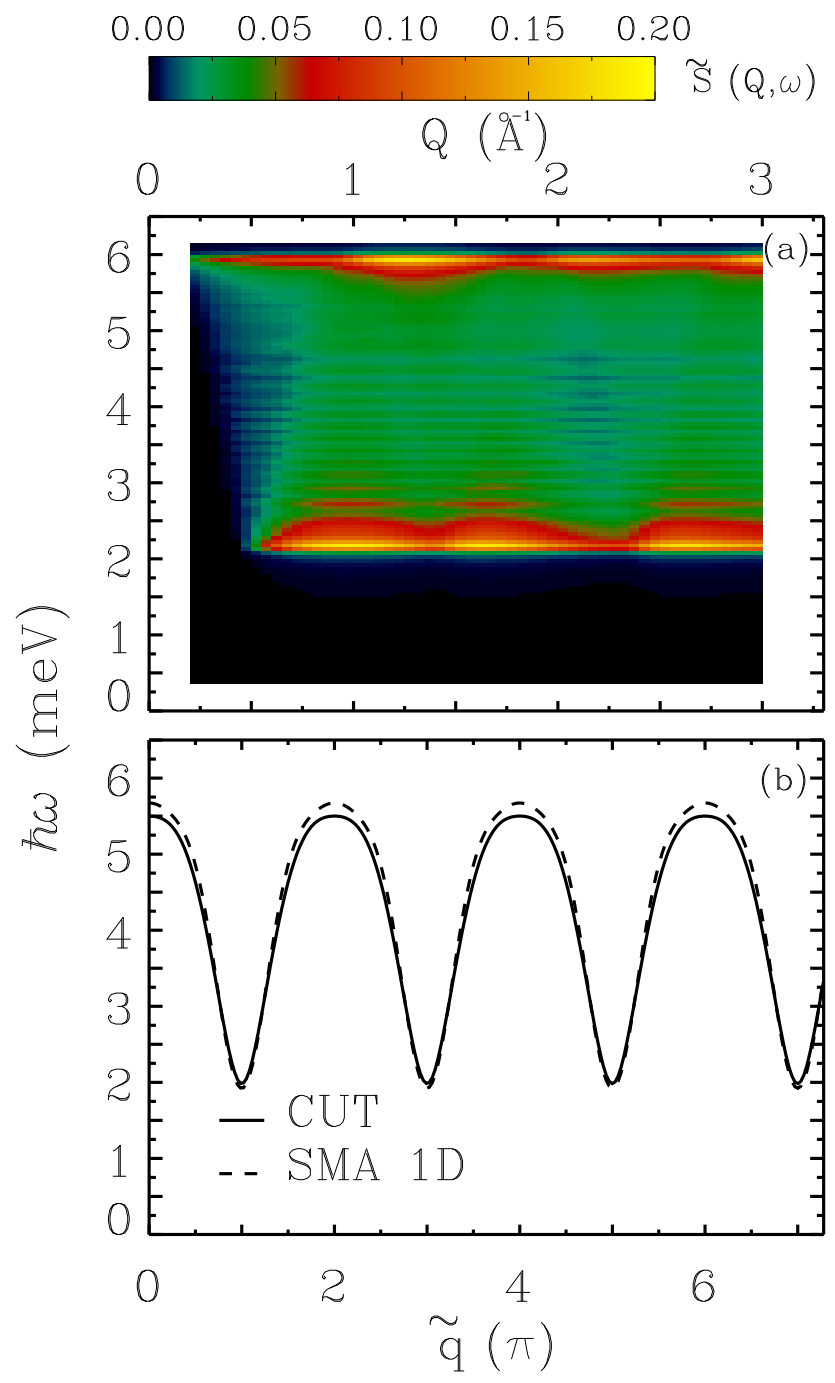

FIG. 10: (a) One-triplon contribution to the powder-averaged dynamic spin correlation function for a spin ladder as calculated by the Continuous Unitary Transformation. Lattice spacings are those appropriate for $\mathrm{Cu}$ (quinoxaline) $\mathrm{Br}_{2}$ and the exchange constants were derived by fitting to neutron scattering data as described in the text. (b) The comparison of triplon dispersion relation for the best fit between CUT (solid line) and SMA (dashed line) ladder models.

are well described by the Continuous Unitary Transformation theory of a spin- $1 / 2$ ladder along the $\mathbf{b}$-direction of $\mathrm{Cu}$ (Quinoxaline) $\mathrm{Br}_{2}$ with exchange constants $J_{\|}=$ $1.98 \mathrm{meV}$ and $J_{\perp}=3.3 \mathrm{meV}$. There is also evidence of inter-ladder dispersion with a bandwidth of approximately $0.2 \mathrm{meV}$. A c-axis alternating chain model was shown to be inconsistent with the data. While there may exist other models that are consistent with the present powder data, our results strongly support the interpretation of $\mathrm{Cu}$ (Quinoxaline) $\mathrm{Br}_{2}$ as a quantum spin- $1 / 2$ ladder with significant inter-rung coupling $\left(J_{\|} / J_{\perp}=0.6\right)$. Efforts are now under way to test this conclusion and ex- plore two-triplon dynamics in this interesting quantum

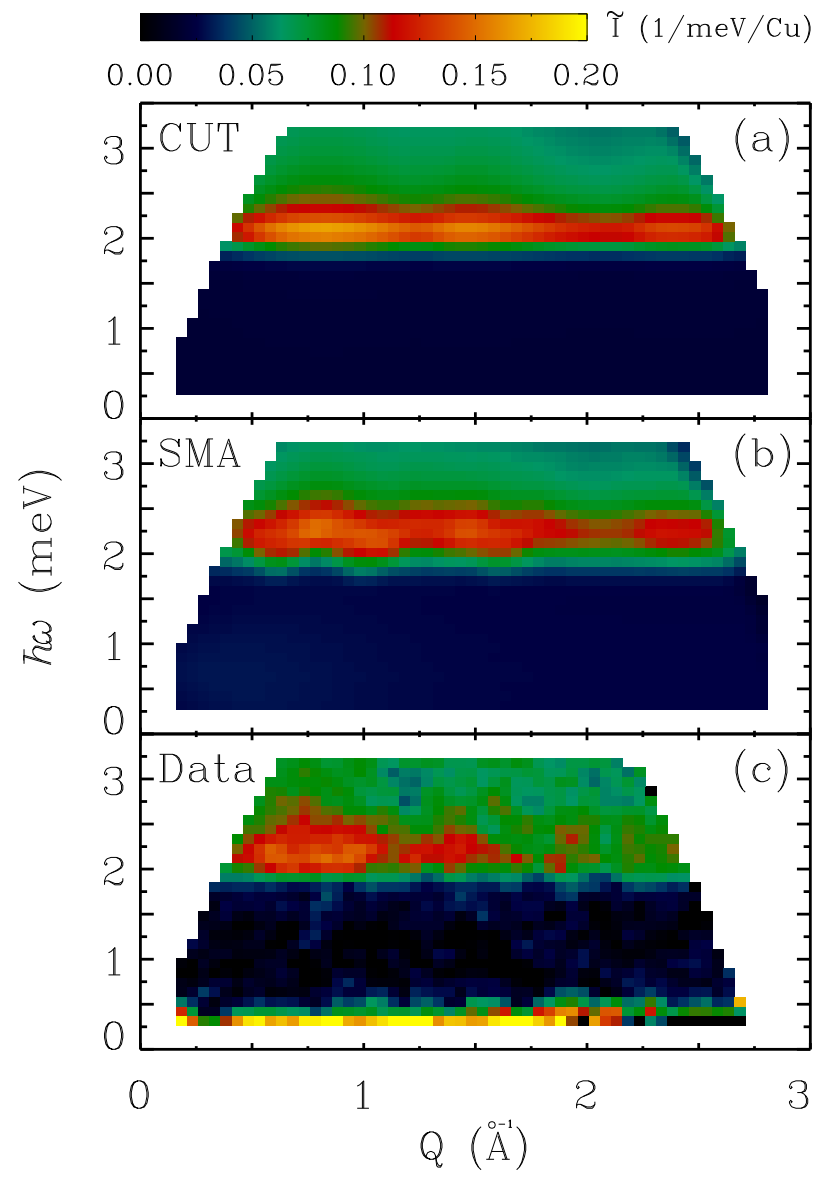

FIG. 11: Comparison of inelastic magnetic neutron scattering $\widetilde{I}(Q, \omega)$ from $\mathrm{Cu}$ (Quinoxaline) $\mathrm{Br}_{2}$ at $\mathrm{T}=1.4 \mathrm{~K}$ (c) to (a) the One-Triplon scattering derived from the Continuous Unitary Transformation (b) a quasi-one-dimensional spin ladder model in the Single-Mode Approximation. The models were complemented with incoherent elastic double scattering, according to Eq. (8) with $\mathcal{T}=0.40$ and an overall additive constant was fit to account for any discrepancies in background subtraction.

paramagnet through inelastic neutron scattering from an assembly of small crystalline samples.

\section{Acknowledgments}

It is a pleasure to thank Rick Paul for help with neutron activation analysis and Seunghun Lee for providing software to implement the detailed balance correction. Work at JHU was supported by the NSF through Grant No. DMR-0306940. This work utilized neutron research facilities that were partially supported by NSF through DMR-0086210 and DMR-0454672. 
1 M. B. Stone, D. H. Reich, C. Broholm, K. Lefmann, C. Rischel, C. P. Landee, and M. M. Turnbull, Phys. Rev. Lett., 91, 037205 (2003).

2 M. Kenzelmann, Y. Chen, C. Broholm, D. H. Reich, and Y. Qiu, Phys. Rev. Lett. 93, 017204 (2004).

${ }^{3}$ E. H. Lieb, T. D. Schultz, and D. C. Mattis, Ann. Phys. (N.Y) 16, 407 (1961).

4 T. Barnes, E. Dagotto, J. Riera, and E. S. Swanson, Phys. Rev. B 47, 3196 (1993).

${ }^{5}$ G. S. Uhrig and H. J. Schulz, Phys. Rev. B 54, R9624 (1996); Erratum 58, 2900 (1998).

6 O. P. Sushkov and V. N. Kotov, Phys. Rev. Lett. 81, 1941 (1998).

7 K. Damle and S. Sachdev, Phys. Rev. B 57, 8307 (1998).

8 C. Jurecka and W. Brenig, Phys. Rev. B 61, 14307 (2000).

9 S. Trebst, H. Monien, C. J. Hamer, Z. Weihong, and R. R. P. Singh, Phys. Rev. Lett. 85, 4373 (2000).

10 C. Knetter, K. P. Schmidt, M. Grüninger, and G. S. Uhrig, Phys. Rev. Lett. 87, 167204 (2001).

11 M. Azuma, Z. Hiroi, M. Takano, K. Ishida, and Y. Kitaoka, Phys. Rev. Lett. 73, 3463 (1994).

12 G. Müller, H. Thomas, H. Beck and J. C. Bonner, Phys. Rev. B 24, 1429 (1981).

13 T. Barnes, Phys. Rev. B 67, 024412 (2003)

14 S. A. Carter, B. Batlogg, R. J. Cava, J. J. Krajewski, W. F. Peck, Jr., and T. M. Rice, Phys. Rev. Lett. 77, 1378 (1996).

15 B. Chiari, O. Piovesana, T. Tarantelli, and P. F. Zanazzi, Inorg. Chem. 29, 1172 (1990).

16 P. R. Hammar and D. H. Reich, J. Appl. Phys. 79, 5392 (1996).

17 G. Chaboussant, P. A. Crowell, L. P. Lévy, O. Piovesana, A. Madouri, and D. Mailly, Phys. Rev. B 55, 3046 (1997).

18 G. Chaboussant, M. -H. Julien, Y. Fagot-Revurat, L. P. Lévy, C. Berthier, M. Horvatic, and O. Piovesana, Phys. Rev. Lett. 79, 925 (1997).

19 P. R. Hammar, D. H. Reich, and C. Broholm, and F. Trouw, Phys. Rev. B 57, 7846 (1997).

20 D. C. Johnston, J. W. Johnson, D. P. Goshorn, and A. J. Jacobson, Phys. Rev. B 35, 219 (1987).

21 R. S. Eccleston, T. Barnes, J. Brody, and J. W. Johnson, Phys. Rev. Lett. 73, 2626 (1994).

22 M. B. Stone, Y. Chen, J. Rittner, H. Yardimci, D. H. Reich, C. Broholm, D. V. Ferraris, and T. Lectka, Phys. Rev. B 65, 64423 (2002)

23 A. W. Garrett, S. E. Nagler, T. Barnes, and B.C. Sales, Phys. Rev. B 55, 3631 (1997).

24 D. A. Tennant, S. E. Nagler, A. W. Garrett, T. Barnes, and C. C. Torardi, Phys. Rev. Lett. 78, 4998 (1997).

25 A. W. Garrett, S. E. Nagler, D. A. Tennant, B. C. Sales, and T. Barnes, Phys. Rev. Lett. 79, 745 (1997).
26 B. R. Patyal, B. L. Scott, and R. D. Willett, Phys. Rev. B 41, 1657 (1991); B. C. Watson, V. N. Kotov, M. W. Meisel, D. W. Hall, G. E. Granroth, W. T. Montfrooij, S. E. Nagler, D. A. Jensen, R. Backov, M. A. Petruska, G. E. Fanucci, and D. R. Talham, Phys. Rev. Lett. 86, 5168 (2001).

27 C. P. Landee, A. Delcheva, C. Galeriu, G. Pena, M. M. Turnbull, and R.D. Willett, Polyhedron 22, 2325 (2003).

28 P. Lumme, S. Lindroos, and E. Lindell, Acta Cryst. C 43, 2053 (1987).

29 Y. Sasago, K. Uchinokura, A. Zheludev, and G. Shirane, Phys. Rev. B 55, 8357 (1997).

30 G. Xu, C. Broholm, D. H.Reich, and M.A. Adams, Phys. Rev. Lett. 84, 4465 (2000).

31 N. Cavadini, G. Heigold, W. Henggeler, A. Furrer, H. U. Güdel, K. Krämer, and H. Mutka, Phys. Rev. B 63, 172414 (2001).

32 N. Cavadini, W. Henggler, A. Furrer, H. -U. Güdel, K. Krämer, and H. Mutka, Eur. Phys. J. B 7, 519 (1999).

33 N. Cavadini, G. Heigold, W. Henggler, A. Furrer, H. U. Güdel, K. Krämer, and H. Mutka, J. Phys.: Condens. Matter 12, 5463 (2000).

34 J. R. D. Copley and J. C. Cook, Chem. Phys. 292, 477 (2003).

35 S. W. Lovesey, "Theory of Thermal Neutron Scattering from Condensed Matter", Clarendon Press, Oxford (1984).

36 Multiple scattering effects can be reduced by using an annular sample geometry and introducing horizontal neutron absorbing plates in the sample.

37 International Tables for Crystallography C, edited by A. J. C. Willson (Kluwer Academic Publishers, Dordrecht/Boston/London, 1995).

38 S. Shamoto, M. Sato, J. M. Tranquada, B. J. Sternlieb, and G. Shirane, Phys. Rev. B 48, 13817 (1993).

39 B. S. Shastry, and B. Sutherland, Phys. Rev. Lett. 47, 964 (1981).

40 K. P. Schmidt and G. S. Uhrig, Phys. Rev. Lett. 90, 227204 (2003).

41 P. C. Hohenberg and W. F. Brinkman, Phys. Rev. B 10, 128 (1974).

42 M. Reigrotzki, H. Tsunetsugu, and T. M. Rice, J. Phys.: Condens. Matter 6, 9235 (1994).

43 C. Knetter and G. S. Uhrig, Eur. Phys. J. B 13, 209 (2000).

44 C. Knetter, K. P. Schmidt, and G. S. Uhrig, J. Phys. A: Math. Gen. 36, 7889 (2003).

45 C. Knetter, K. P. Schmidt, and G. S. Uhrig, Eur. Phys. J. B 36, 525 (2004).

46 K. P. Schmidt, C. Knetter, and G. S. Uhrig, Acta Physica Polonica B 34, 1481 (2003).

47 K. P. Schmidt, H. Monien, and G. S. Uhrig, Phys. Rev. B 67, 184413 (2003). 